\title{
High Bandwidth Atomic Magnetometery with Continuous Quantum Non-demolition Measurements
}

\author{
V. Shah, G. Vasilakis and M. V. Romalis \\ Department of Physics, Princeton University, Princeton, New Jersey 08544, USA
}

\begin{abstract}
We describe an experimental study of spin-projection noise in a high sensitivity alkali-metal magnetometer. We demonstrate a four-fold improvement in the measurement bandwidth of the magnetometer using continuous quantum non-demolition (QND) measurements. Operating in the scalar mode with a measurement volume of $2 \mathrm{~cm}^{3}$ we achieve magnetic field sensitivity of $22 \mathrm{fT} / \mathrm{Hz}^{1 / 2}$ and a bandwidth of $1.9 \mathrm{kHz}$ with a spin polarization of only $1 \%$. Our experimental arrangement is naturally back-action evading and can be used to realize sub-fT sensitivity with a highly polarized spin-squeezed atomic vapor.
\end{abstract}

The limits imposed by quantum mechanics on precision measurements have been the subject of long-standing interest. They are particularly important in atomic systems that form the basis of leading frequency standards, magnetometers and inertial sensors. The Heisenberg uncertainty principle imposes a limit on measurement sensitivity with uncorrelated atoms known as the standard quantum limit (SQL). One can improve upon this limit using spin squeezing techniques [1]. However, it has been shown theoretically that in the presence of a constant rate of decoherence spin squeezing does not lead to a significant improvement of the long term measurement sensitivity, but can be used to reduce the measurement time [2-4]. Such an increase in the measurement bandwidth without loss of sensitivity is particularly important for systems with long spin coherence times, which can achieve the highest measurement resolution but often require impractically-long interrogation times.

Here we study the limits imposed by quantum spin fluctuations in a dense hot alkali-metal vapor used in sensitive atomic magnetometers [5]. While the sensitivity of most previous atomic magnetometers has been limited by photon shot noise or technical noise, we investigate the regime limited by spin projection noise. We show that in this regime the measurement bandwidth can be increased using quantum-non-demolition paramagnetic Faraday rotation measurements [6] without loss of sensitivity. We point out that the bandwidth increase can be realized with only a weak squeezing condition $\left(\Delta J_{x}\right)^{2}<J / 2$ for a system with total spin $J$, and demonstrate experimentally an increase in the magnetometer bandwidth from $420 \mathrm{~Hz}$ to $1.9 \mathrm{kHz}$. Increasing the measurement bandwidth is important for many applications of atomic magnetometery, such as detection of biological magnetic fields from the heart and the brain [7, 8] and nuclear magnetic resonance 9 11], where the bandwidth of magnetic signals often exceeds the natural bandwidth of the atomic magnetometers. We operate the magnetometer in a scalar measurement mode and obtain magnetic field sensitivity of $22 \mathrm{fT} / \mathrm{Hz}^{1 / 2}$, in agreement with theoretical prediction for the size of spin projection noise and only a factor of 2 away from the best sensitivity previously ob-

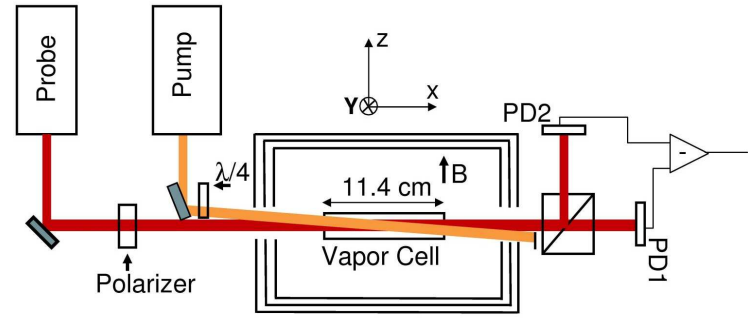

FIG. 1: Apparatus for high-bandwidth QND scalar magnetometry.

tained in a scalar magnetometer 12. Our measurements are performed with spin polarization of only $1 \%$, where back-action evasion is not necessary. However, our measurement configuration is naturally back-action evading and thus much higher sensitivity can be expected in a highly-polarized atomic vapor.

The experimental geometry is shown in Fig. 1. A Pyrex glass cell $1 \mathrm{~cm}$ in diameter and $11.4 \mathrm{~cm}$ long contains enriched ${ }^{87} \mathrm{Rb}$ and 60 torr of Nitrogen buffer gas and is heated to about $110^{\circ} \mathrm{C}$. The cell is placed inside multilayer magnetic shields and a magnetic field of $4.4 \mu \mathrm{T}$ generated by an ultra-stable current source is directed perpendicular to the long axis of the cell, giving a Larmor frequency of $31 \mathrm{kHz}$. A linearly polarized probe beam detuned from the D1 line is directed along the length of the cell. Paramagnetic Faraday rotation induced by the atoms is measured with a balanced polarimeter.

First we measure the spin-projection noise in an unpolarized vapor. The power spectrum of the polarimeter output is plotted in Fig. 2, showing a large noise peak centered at the Larmor frequency. Detection of alkalimetal spin fluctuations by paramagnetic Faraday rotation was first demonstrated by Alexandrov [13] and later studied in 14 16. We obtain a ratio of the peak spin noise power to the flat photon shot noise background equal to 22 , substantially higher than in previous experiments. For our conditions the probe laser is detuned far away relative to the excited state hyperfine structure and the Lorentzian and Doppler linewidths, but comparable 
to the ground-state hyperfine splitting. The optical rotation angle in this case is proportional to the vector spin polarization, with negligible contribution from the tensor polarization [17, 18]. For the D1 line the rotation angle is given by

$$
\phi=\frac{c r_{e} f_{o s c} n l}{(2 I+1)}\left[D\left(\nu-\nu_{a}\right)\left\langle F_{x}^{a}\right\rangle-D\left(\nu-\nu_{b}\right)\left\langle F_{x}^{b}\right\rangle\right],
$$

where $r_{e}=2.82 \times 10^{-13} \mathrm{~cm}$ is the classical electron radius, $f_{\text {osc }}=0.34$ is the oscillator strength of the D1 transition in $\mathrm{Rb}, n$ is the vapor density of alkali-metal atoms, $l$ is the length of the cell along the probe direction, $\left\langle F_{x}^{a}\right\rangle$ and $\left\langle F_{x}^{b}\right\rangle$ are the expectation values of the atomic $\operatorname{spin} \mathbf{F}=\mathbf{I}+\mathbf{S}$ in the $a=I+1 / 2$ and $b=I-1 / 2$ hyperfine states with optical resonance frequencies of $\nu_{a}$ and $\nu_{b}$. Here $D(\nu)$ is the dispersion profile given by $D(\nu)=\left(\nu-\nu_{0}\right) /\left[\left(\nu-\nu_{0}\right)^{2}+(\Delta \nu / 2)^{2}\right]$, where $\Delta \nu$ is the Lorentzian FWHM due to pressure broadening. For unpolarized, uncorrelated atoms the r.m.s. spin fluctations are given by

$$
\sqrt{\overline{\left\langle F_{x}^{a}\right\rangle^{2}}}=\sqrt{\frac{F^{a}\left(F^{a}+1\right)\left(2 F^{a}+1\right)}{6(2 I+1) N}},
$$

where $N$ is the number of atoms being probed, and similar for $F_{x}^{b}$. Most spin relaxation mechanisms in alkalimetal vapor affect only the electron spin and hence can introduce correlations between $F_{x}^{a}$ and $F_{x}^{b}$. However, in the regime when the Larmor frequency $\omega$ is much larger than the spin relaxation rate, the transverse spin components of $F^{a}$ and $F^{b}$ precess in opposite directions, quickly destroying any correlations. Hence polarization rotation noise from the two terms in Eq. (1) is not correlated. The number of atoms participating in the measurement depends on the transverse intensity profile of the probe beam $I(y, z)$ and is determined by summing noise variance from different parts of the beam,

$$
N=n l \frac{\left[\int I(y, z) d y d z\right]^{2}}{\int I(y, z)^{2} d y d z} .
$$

The data in Fig. 2 are very well described by a single Lorentzian and a constant photon shot noise background. The Lorentzian half-width of the noise peak is equal to $340 \mathrm{~Hz}$, with about half of the width due to spin-exchange relaxation and the rest due to absorption of the probe beam and diffusion. The flat noise background is in agreement with expected level of photon shot noise. As discussed in [14 16], the noise spectrum in general is a sum of Lorentzians, but the widths of the resonances for $\left\langle F_{x}^{a}\right\rangle$ and $\left\langle F_{x}^{b}\right\rangle$ are similar and the strength of the $\left\langle F_{x}^{b}\right\rangle$ signal is smaller, so it is difficult to distinguish them. There are only small hints of a deviation from a Lorentzian in the wings of the noise peak. The total r.m.s. noise $\phi_{r m s}$ given by the area under the peak of the

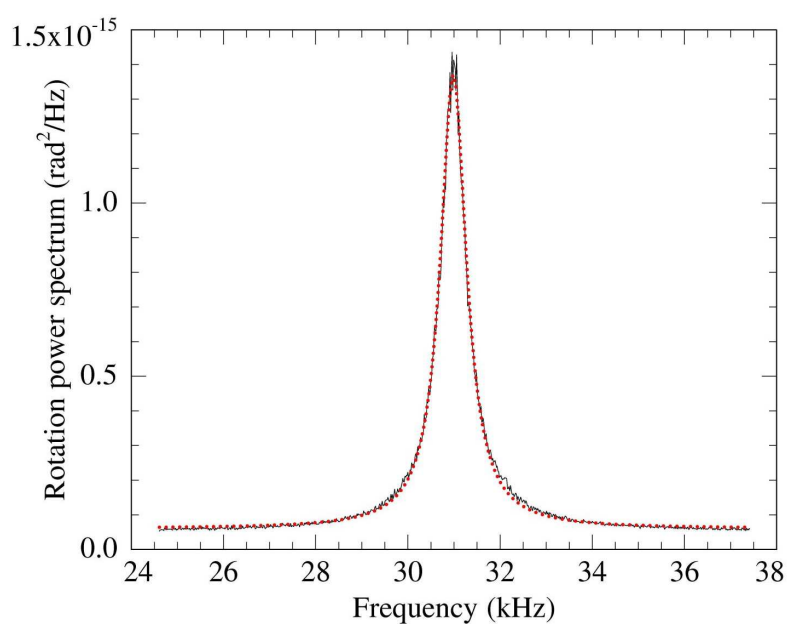

FIG. 2: Measured spin noise power spectrum of unpolarized atoms (solid line) with a fit to a Lorentzian plus flat photon shot noise background (dashed line). The averaging time for the noise spectrum is $350 \mathrm{~s}$.

power spectrum is not affected by the shape of the spectrum. Diffusion of atoms in and out of the probe beam also distorts the spectrum of the noise from a Lorentzian shape, causing a sharper peak due to Ramsey narrowing [19], but it also does not change $\phi_{r m s}$.

For the data presented in Fig. 2 the density of ${ }^{87} \mathrm{Rb}$ atoms is equal $n=8.7 \times 10^{12} \mathrm{~cm}^{-3}$, determined by a measurement of the transverse spin relaxation time due to spin exchange collisions. The intensity profile of the probe beam was measured in both directions by the scanning edge technique and had effective dimensions of $3.8 \times 4.5 \mathrm{~mm}^{2}$. We find that the effective number of atoms being probed is $N=1.7 \times 10^{13}$. The probe laser is detuned by $19 \mathrm{GHz}$ from the $F=2$ state and $25.8 \mathrm{GHz}$ from $F=1$ state, much larger than the Lorentzian optical FWHM $\Delta \nu=1.42 \mathrm{GHz}$. According to Eq (1), this gives $\phi_{r m s}^{t h}=1.07 \times 10^{-6} \mathrm{rad}$. Experimentally, the area under the spin noise peak is equal $\phi_{r m s}^{\exp }=1.19 \times 10^{-6} \mathrm{rad}$. The agreement at the $10 \%$ level is quite good given the uncertainty in the number of atoms participating in the measurement. Using a different cell with natural abundance of $\mathrm{Rb}$ isotopes we verified experimentally that the ratio of spin noise for ${ }^{87} \mathrm{Rb}$ and ${ }^{85} \mathrm{Rb}$ isotopes, which have different nuclear spins, is consistent with our analysis within a few percent. In a previous detailed study of atomic spin noise [15] the overall level of spin noise was off by a factor of 2 from predictions.

A scalar atomic magnetometer measuring the absolute value the magnetic field is realized using a circularly polarized pump beam propagating nearly parallel to the probe beam but missing the photodetectors. We use BellBloom excitation [20] of the spin precession around $B_{z}$ field by sinusoidally modulating the current in a DFB laser used to generate the pumping light at the Larmor 


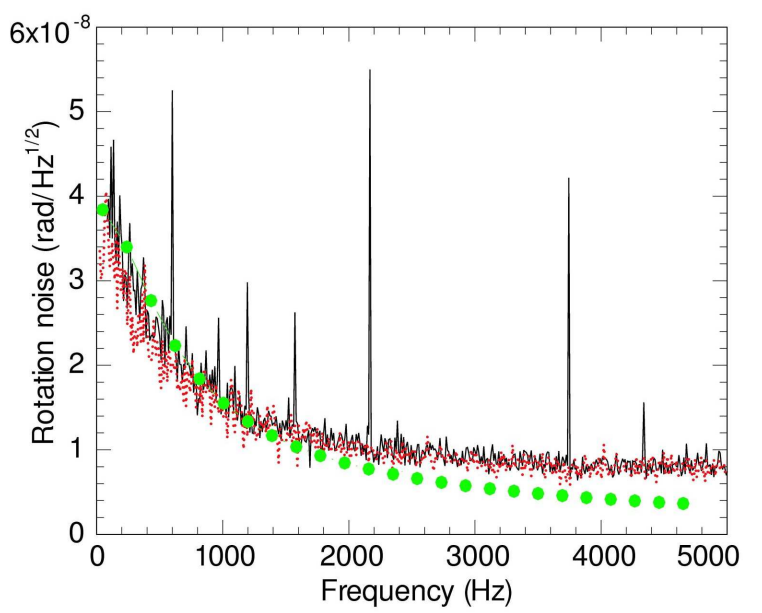

FIG. 3: Lock-in output amplitude noise spectrum for polarized atoms (solid line) and unpolarized atoms (dashed line). The signal expected in the magnetometer for a $22 \mathrm{fT}_{\text {rms }}$ magnetic field is shown with dots. The spikes in the noise spectrum for polarized atoms are from technical magnetic noise.

frequency. The polarimeter signal is directly digitized using a fast, high resolution A/D card and lock-in demodulation of the data is implemented at the analysis stage. The noise spectrum of the out-of-phase component of the lock-in signal, proportional to small variations of $B_{z}$ field, is shown in Fig. 3 with and without the pump beam. The intensity of the pump beam is increased until the noise level just starts to increase due to technical noise sources and is equal to about $100 \mu \mathrm{W}$. The spin polarization of the atomic vapor, determined from the amplitude of the oscillating rotation signal, is equal to $1.0 \%$. Discrete noise peaks from magnetic interference can be seen for polarized atoms. The magnetic field sensitivity of the rotation signal is calibrated by applying a known modulation to $B_{z}$ field at various frequencies. We show in Fig. 3 with filled circles the expected magnetometer signal for a $22 \mathrm{fT}_{r m s}$ oscillating magnetic field as a function of frequency. It can be seen that at higher frequencies the response of the magnetometer drops, but the noise level decreases as well, so the magnetometer retains its sensitivity up to much higher frequencies than the resonance linewidth.

In fact, the sensitivity remains constant as long as the noise spectrum is dominated by spin noise. This can be easily seen in the simpler case of a spin- $1 / 2$ system when the magnetic resonance is described by Bloch equations. One can show that the absolute value of the magnetometer signal in response to an oscillating $B_{z}$ field decreases with frequency as the square root of a Lorentzian with a width given by the inverse of the spin coherence time $T_{2}$,

$$
S(f)=S_{0} /\left[1+\left(2 \pi f T_{2}\right)^{2}\right]^{1 / 2} .
$$

The shape of the spin noise spectrum is also described by the square root of a Lorentzian with the same width

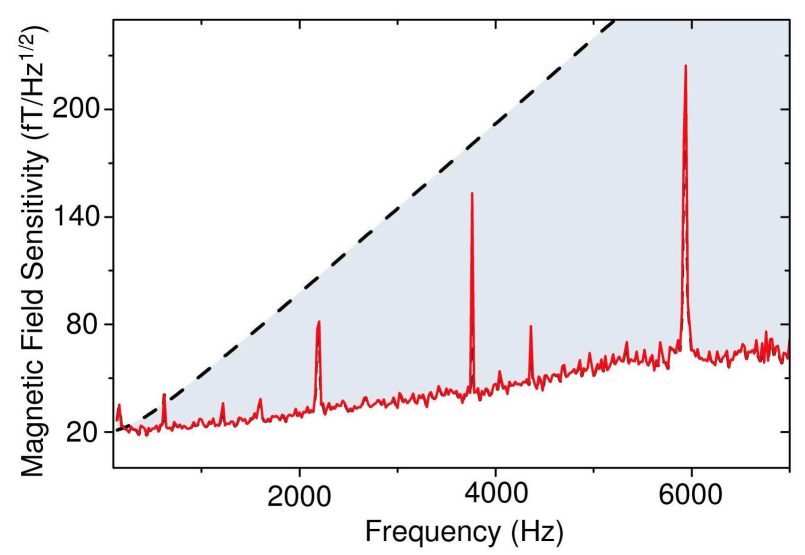

FIG. 4: Experimentally observed magnetic field noise spectral density corrected for the frequency response of the magnetometer (solid line). The spikes are due to narrow-band magnetic noise. Dashed line shows expected magnetometer sensitivity for a demolition measurement assuming a flat noise spectrum. The shaded area represents improvement in the magnetometer sensitivity at high frequencies as a result of QND measurements. For QND measurements the sensitivity decreases by $\sqrt{2}$ at $1.9 \mathrm{kHz}$.

[21]. Hence the signal and the noise decrease simultaneously, maintaining constant sensitivity. In Fig. 4 we show the magnetic field sensitivity of the magnetometer as a function of frequency by dividing the noise spectrum by the response curve. The sensitivity remains nearly constant up to $2 \mathrm{kHz}$, while the resonance linewidth of the magnetometer is equal to $420 \mathrm{~Hz}$ in this case. The increase in the bandwidth is a direct result of the non-white nature of the spin noise that is realized with QND measurements which preserve temporal correlations of the spin expectation value. In contrast, if the spin polarization is monitored using optical absorption instead of Faraday rotation, or if the optical density of the vapor on resonance is less than one, the noise spectrum would be white. We show in Fig. 4 the sensitivity that would be obtained with such a flat noise spectrum for comparison. This demonstrates the role of quantum-non-demolition measurements, they do not improve the performance at low frequencies but increase the bandwidth of measurements without any penalty in sensitivity. The necessary condition for bandwidth increase can be written as $\left(\Delta J_{x}\right)^{2}<J / 2$ in order to resolve temporal spin correlations. This is a weaker condition than $\left(\Delta J_{x}\right)^{2}<\left\langle J_{z}\right\rangle / 2$ that usually defines spin squeezing [1].

In general, one can define the measurement bandwidth of the magnetometer as the frequency at which the sensitivity of the magnetometer drops by a factor of $1 / \sqrt{2}$ from its value at low frequency. For a square root of Lorentzian frequency response and a flat noise spectrum $f_{B W}=1 /\left(2 \pi T_{2}\right)$. Using electronic feedback or self-oscillating operation it is possible to obtain a flat frequency response, however, even in this case the sensi- 
tivity of the magnetometer becomes worse at frequencies higher than $1 / T_{2}$ because of noise induced by the feedback [22]. For a quantum non-demolition paramagnetic Faraday rotation measurement with a far-detuned probe laser, the spectral noise density can be written as

$$
\phi_{n}(f)=\frac{1}{\sqrt{2 \Phi}}\left\{\frac{1}{\eta}+\frac{N_{a b} \Gamma_{p r} T_{2} \beta}{1+\left[2 \pi\left(f-f_{0}\right) T_{2}\right]^{2}}\right\}^{1 / 2},
$$

where $\Phi$ is the probe laser photon flux, $\eta$ is the quantum efficiency of the photodetectos, $N_{a b}$ is the number of absorption lengths (optical density) of the atomic sample on resonance, $\Gamma_{p r}$ is the optical pumping rate of the probe laser and $\beta$ is a factor of order unity depending on the nuclear spin and polarization of the atomic ensemble $(\beta=1$ for $I=0)$. Using Eq. (4) and (5) it's easy to show that the bandwidth of the magnetometer with quantum-non-demolition measurements is given by $f_{B W}=\left(\eta N_{a b} \Gamma_{p r} T_{2} \beta+1\right)^{1 / 2} /\left(2 \pi T_{2}\right)$. In the regime where the spin relaxation rate is dominated by the optical pumping rate of the probe laser, $\Gamma_{p r} T_{2} \simeq 1$, the bandwidth is increased by a factor on the order of the square root of the optical density on resonance $N_{a b}^{1 / 2}$.

For our conditions the back-action of the probe beam is not significant because of low spin polarization. However, our experimental arrangement is naturally back-action evading and can be used to generate conditional spin squeezing. In the regime of far deturning the back-action of the probe beam is due to the light shift created by the quantum fluctuations of the circular polarization of the probe beam and is equivalent to a fictitious magnetic field parallel to the propagation direction of the probe beam [17]. Our probe beam is directed perpendicular to a large static magnetic field and thus the light shift fluctuations only contribute in second order to the absolute value of the magnetic field measured by the scalar magnetometer. A fluctuating magnetic field along the $x$ direction will generate a small $z$ component of the polarization that is not directly measured. Therefore this arrangement can be used to generate conditional spin squeezing in a highly polarized vapor [23].

The magnetic field sensitivity of $22 \mathrm{fT} / \mathrm{Hz}^{1 / 2}$ obtained in this experiment is the best measured sensitivity with a single-channel scalar magnetometer and within a factor of 2 of the best measured sensitivity obtained in a gradiometer arrangement [12]. Scalar atomic magnetometers have lagged in sensitivity compared to other types of atomic magnetometers [5] because of spin-exchange broadening. The measurement volume used in our sensor is about $2 \mathrm{~cm}^{3}$ and the spin polarization is equal to $1 \%$. The sensitivity of the magnetometer will be optimized if the intensity of the pumping light is increased to obtain spin polarization of $50 \%$ with a factor of 2 broadening of the magnetic resonance. The signal will increase by a factor of 50 , while the spin-projection noise will not change appreciably, resulting in a magnetic field sensitivity of about $0.6 \mathrm{fT} / \mathrm{Hz}^{1 / 2}$, consistent with spin-exchange limited sensitivity for alkali-metal magnetometers [12]. Alternatively, operating in the regime of low spin polarization can be advantageous if it is desired to minimize the heading errors of the magnetometer which depend on the degree of polarization [24].

In conclusion, we investigated operation of an atomic magnetometer in the spin-projection noise limited regime. The magnitude of the spin noise is in good agreement with theory. We demonstrated an increase in the magnetometer bandwidth by a factor of 4 using quantum non-demolition measurements. Such increase of the measurement bandwidth without loss of sensitivity is important for many practical applications of atomic magnetometery, such as detection of NMR and biological fields. Similar QND measurements can also be used to increase the bandwidth of magnetometers based on nuclear spins with very long spin coherence times and the update rate of atomic clocks based on very narrow transitions. This work was supported by an ONR MURI award.

[1] D. J. Wineland, J. J. Bollinger, W. M. Itano, F. L. Moore, and D. J. Heinzen, Phys. Rev. A 46, R6797 (1992).

[2] S. F. Huelga, C. Macchiavello, T. Pellizzari, A. K. Ekert, M. B. Plenio, and J. I. Cirac, Phys. Rev. Lett. 79, 3865 (1997).

[3] A. André, A. S. Sørensen, and M. D. Lukin, Phys. Rev. Lett. 92, 230801 (2004).

[4] M. Auzinsh, D. Budker, D. F. Kimball, S. M. Rochester, J. E. Stalnaker, A. O. Sushkov, and V. V. Yashchuk, Phys. Rev. Lett. 93, 173002 (2004).

[5] D. Budker and M. Romalis, Nature Physics 3, 227 (2007).

[6] Y. Takahashi, K. Honda, N. Tanaka, K. Toyoda, K. Ishikawa, and T. Yabuzaki, Phys. Rev. A 60, 4974 (1999).

[7] G. Bison, R. Wynands, and A. Weis, Optics Express 11, 904 (2003).

[8] H. Xia, A. B.-A. Baranga, D. Hoffman, and M. V. Romalis, Appl. Phys. Lett. 89 (2006).

[9] I. M. Savukov and M. V. Romalis, Phys. Rev. Lett. 94, 123001 (2005).

[10] S. Xu, V. V. Yashchuk, M. H. Donaldson, S. M. Rochester, D. Budker, and A. Pines, Proc. Nat. Acad. Sci. 103, 12668 (2006).

[11] M. P. Ledbetter, I. M. Savukov, D. Budker, V. Shah, S. Knappe, J. Kitching, D. J. Michalak, S. Xu, and A. Pines, Proc. Nat. Acad. Sci. 105, 2286 (2008).

[12] S. J. Smullin, I. M. Savukov, G. Vasilakis, R. K. Ghosh, and M. V. Romalis, Phys. Rev. A 80, 033420 (2009).

[13] E. Alexsandrov and V. Zapassky, Zh. Eksp. Teor. Fiz. 81, 132 (1981).

[14] S. Crooker, D. Rickel, A. Balatsky, and D. Smith, Nature 431, 49 (2004).

[15] B. Mihaila, S. A. Crooker, D. G. Rickel, K. B. Blagoev, P. B. Littlewood, and D. L. Smith, Phys. Rev. A 74, 043819 (2006).

[16] G. E. Katsoprinakis, A. T. Dellis, and I. K. Kominis, Phys. Rev. A 75, 042502 (2007).

[17] B. S. Mathur, H. Tang, and W. Happer, Phys. Rev. 171, 
$11(1968)$

[18] J. M. Geremia, J. K. Stockton, and H. Mabuchi, Phys. Rev. A 73, 042112 (2006).

[19] Y. Xiao, I. Novikova, D. F. Phillips, and R. L. Walsworth, Phys. Rev. Lett. 96, 043601 (2006).

[20] W. E. Bell and A. L. Bloom, Phys. Rev. Lett. 6, 280 (1961).

[21] M. Braun and J. König, Phys. Rev. B 75, 085310 (2007).
[22] P. Schwindt, L. Hollberg, and J. Kitching, Rev.Sci. Instrum. 76 (2005).

[23] A. Kuzmich, L. Mandel, and N. P. Bigelow, Phys. Rev. Lett. 85, 1594 (2000)

[24] S. J. Seltzer, P. J. Meares, and M. V. Romalis, Phys. Rev. A 75, 051407 (2007). 